\title{
A note on the regularity of solutions of Hamilton-Jacobi equations with superlinear growth in the gradient variable
}

\author{
P. Cardaliaguet*
}

October 25, 2018

\section{Introduction}

We investigate the regularity of solutions to the Hamilton-Jacobi equation

$$
\begin{cases}-u_{t}(x, t)+b(x, t)|D u(x, t)|^{q}+f(x, t) \cdot D u(x, t)=0 & \text { in } \mathbb{R}^{N} \times(0, T) \\ u(x, T)=g(x) & \text { for } x \in \mathbb{R}^{N}\end{cases}
$$

under the following assumptions:

$$
q>1
$$

$b: \mathbb{R}^{N} \times(0, T) \rightarrow \mathbb{R}, f: \mathbb{R}^{N} \times(0, T) \rightarrow \mathbb{R}^{N}$ and $g: \mathbb{R}^{N} \rightarrow \mathbb{R}$ are continuous and bounded by some constant $M$,

$$
b(x, t) \geq \delta>0 \quad \forall(x, t) \in \mathbb{R}^{N} \times(0, T)
$$

for some $\delta>0$.

Regularity of solutions of Hamilton-Jacobi equations with superlinear growth have been the object of several works (see in particular Lions [6], Barles [3], Rampazzo, Sartori [7]). Our aim is to show that $u$ is locally Hölder continuous with Hölder exponent and constant depending only $M, \delta$, $q$ and $T$. What is new compared to the previous works is that the regularity does not depend on the smoothness of the maps $b, f$ and $g$, but only on the growth condition. The motivation for this is the homogenization of

${ }^{*}$ Laboratoire de Mathématiques, UMR 6205, Université de Bretagne Occidentale, 6 Av. Le Gorgeu, BP 809, 29285 Brest. e-mail : Pierre.Cardaliaguet@univ-brest.fr. This work was partially supported by the ANR project MICA. 
Hamilton-Jacobi equations, where such estimates are needed. Here is our result.

Theorem 1.1 There is some constant $\theta=\theta(M, \delta, q, T)$ and, for any $\tau>0$, some constant $K_{\tau}=K(\tau, M, \delta, q, T)$ such that, for any $x_{0}, x_{1} \in \mathbb{R}^{N}$, for any $t_{0}, t_{1} \in[0, T-\tau]$,

$$
\left|u\left(x_{0}, t_{0}\right)-u\left(x_{1}, t_{1}\right)\right| \leq K_{\tau}\left(\left|x_{0}-x_{1}\right|^{(\theta-p) /(\theta-1)}+\left|t_{0}-t_{1}\right|^{(\theta-p) / \theta}\right)
$$

The proof of the result relies on the representation of the solution $u$ of (11) as the value function of a problem of calculus of variations (see [1, [2]): Namely, setting $p=\frac{q}{q-1}$, we have

$$
u(x, t)=\inf \left(\int_{t}^{T} a(x(s), s)\left|f(x(s), s)+x^{\prime}(s)\right|^{p} d s+g(x(T))\right)
$$

where the infimum is taken over the set of functions $x(\cdot) \in W^{1, p}\left([t, T], \mathbb{R}^{N}\right)$ such that $x(t)=x$ and where

$$
a(x, t)=\left(\frac{1}{b(x, t)}\right)^{p-1}\left(p^{-1 /(p-1)}-p^{-p /(p-1)}\right)^{p-1} .
$$

From now on we work on the control representation of the solution $u$. To simplify the notations, we assume without loss of generality that $b$ is also bounded by $M$ and satisfies

$$
a(x, t) \geq \delta>0 \quad \forall(x, t) \in \mathbb{R}^{N} \times(0, T)
$$

The paper is organized as follows. In the first section, we use a kind of reverse Hölder inequality to prove that the optimal solutions of (5) are in some sense slightly "more integrable" than what we could expect. In the second step we show that this integrability implies the desired Hölder regularity for the value function. In Appendix, we prove the reverse Hölder inequality.

Aknowledgement : We wish to thank Guy Barles for useful discussions.

\section{Estimate of the optimal of the controlled system}

The key remark of this section is Lemma 2.5 stating that optimal controls are "more integrable" than what could be expected. This is proved through several steps and the use of a reverse Hölder inequality. 
Lemma 2.1 There is a constant $K \geq 0$ depending only on $M, \delta, p, T$, such that, for any optimal solution $\bar{x}$ of (5) starting from $x_{0}$ at time $t_{0}$, we have

$$
\int_{t_{0}}^{T}\left|\bar{x}^{\prime}(s)\right|^{p} d s \leq K
$$

Proof of Lemma 2.1 : Comparing $\bar{x}$ with the constant solution $\tilde{x}(t)=x_{0}$ we get

$\int_{t_{0}}^{T} a(\bar{x}(s), s)\left|f(\bar{x}(s), s)+\bar{x}^{\prime}(s)\right|^{p} d s+g(\bar{x}(T)) \leq \int_{t_{0}}^{T} a\left(x_{0}, s\right)\left|f\left(x_{0}, s\right)\right|^{p} d s+g\left(x_{0}\right)$

with

$$
\begin{gathered}
g\left(x_{0}\right)-g(\bar{x}(T)) \leq 2 M \\
\int_{t_{0}}^{T} a(\bar{x}(s), s)\left|f(\bar{x}(s), s)+\bar{x}^{\prime}(s)\right|^{p} d s \\
\geq \delta \int_{t_{0}}^{T}\left|f(\bar{x}(s), s)+\bar{x}^{\prime}(s)\right|^{p} d s \\
\geq \frac{\delta}{2^{p-1}}\left(\int_{t_{0}}^{T}\left|\bar{x}^{\prime}(s)\right|^{p} d s-M^{p}\left(T-t_{0}\right)\right)
\end{gathered}
$$

and

$$
\int_{t_{0}}^{T} a\left(x_{0}, s\right)\left|f\left(x_{0}, s\right)\right|^{p} d s \leq M^{p+1}\left(T-t_{0}\right) .
$$

Whence the result with $K=2^{p-1}\left(M^{p+1} T+2 M\right) / \delta+M^{p} T$.

Lemma 2.2 There are some constants $A \geq 1$ and $B \geq 0$ depending only on $M, \delta, p, T$, such that, for any optimal solution $\bar{x}$ of (5) starting from $x_{0}$ at time $t_{0}$, we have

$$
\frac{1}{h} \int_{t_{0}}^{t_{0}+h}\left|\bar{x}^{\prime}(s)\right|^{p} d s \leq A\left(\frac{1}{h} \int_{t_{0}}^{t_{0}+h}\left|\bar{x}^{\prime}(s)\right| d s\right)^{p}+B \quad \forall h \in\left[0, T-t_{0}\right]
$$

Proof of Lemma 2.2 : Let us fix $h \in\left(0, T-t_{0}\right)$ and let us define

$$
\tilde{x}(t)= \begin{cases}\frac{\bar{x}\left(t_{0}+h\right)-x_{0}}{h}\left(t-t_{0}\right)+x_{0} & \text { if } t \in\left[t_{0}, t_{0}+h\right] \\ \bar{x}(t) & \text { otherwise }\end{cases}
$$

Since $\bar{x}$ is optimal and $\tilde{x}(T)=\bar{x}(T)$ we have

$$
\int_{t_{0}}^{t_{0}+h} a(\bar{x}(s), s)\left|f(\bar{x}(s), s)+\bar{x}^{\prime}(s)\right|^{p} d s \leq \int_{t_{0}}^{t_{0}+h} a(\tilde{x}(s), s)\left|f(\tilde{x}(s), s)+\tilde{x}^{\prime}(s)\right|^{p} d s
$$


Then we get the desired result by noticing that

$$
\begin{array}{rl}
\int_{t_{0}}^{t_{0}+h} & a(\bar{x}(s), s)\left|f(\bar{x}(s), s)+\bar{x}^{\prime}(s)\right|^{p} d s \\
& \geq \delta \int_{t_{0}}^{t_{0}+h}\left|f(\bar{x}(s), s)+\bar{x}^{\prime}(s)\right|^{p} d s \\
& \geq \frac{\delta}{2^{p-1}}\left(\int_{t_{0}}^{t_{0}+h}\left|\bar{x}^{\prime}(s)\right|^{p} d s-M^{p} h\right)
\end{array}
$$

and

$$
\begin{array}{rl}
\int_{t_{0}}^{t_{0}+h} & a(\tilde{x}(s), s)\left|f(\tilde{x}(s), s)+\tilde{x}^{\prime}(s)\right|^{p} d s \\
& \leq M \int_{t_{0}}^{t_{0}+h}\left|f(\tilde{x}(s), s)+\tilde{x}^{\prime}(s)\right|^{p} d s \\
& \leq 2^{p-1} M\left(M^{p} h+\int_{t_{0}}^{t_{0}+h}\left|\left(\bar{x}(h)-x_{0}\right) / h\right|^{p} d s\right. \\
& \leq 2^{p-1} M\left(M^{p} h+h^{1-p}\left(\int_{t_{0}}^{t_{0}+h}\left|\bar{x}^{\prime}(s)\right| d s\right)^{p}\right)
\end{array}
$$

In the following Lemma we get rid of the constant $B$ in (77). Assume that $\alpha \in L^{p}\left(\left[t_{0}, T\right], \mathbb{R}^{+}\right)$satisfies

$$
\frac{1}{h} \int_{t_{0}}^{t_{0}+h}|\alpha(s)|^{p} d s \leq A\left(\frac{1}{h} \int_{t_{0}}^{t_{0}+h}|\alpha(s)| d s\right)^{p}+B \quad \forall h \in\left[0, T-t_{0}\right]
$$

Let $z(t)=\int_{t_{0}}^{t} \alpha(s) d s$ and

$$
z_{1}(s)=\max \left\{z(s),\left(\frac{B}{A}\right)^{1 / p}\left(s-t_{0}\right)\right\} \quad \forall s \in\left[t_{0}, T\right] .
$$

Set $\alpha_{1}(t)=z_{1}^{\prime}(t)$. We note for later use that $z_{1}(t) \geq z(t)$ on $\left[t_{0}, T\right]$ and that, if $z_{1}(t)=z(t)$, then $\int_{t_{0}}^{t}\left(\alpha_{1}(s)\right)^{p} d s \leq \int_{t_{0}}^{t}(\alpha(s))^{p} d s$. We claim:

\section{Lemma 2.3}

$$
\frac{1}{h} \int_{t_{0}}^{t_{0}+h}\left|\alpha_{1}(s)\right|^{p} d s \leq 2 A\left(\frac{1}{h} \int_{t_{0}}^{t_{0}+h}\left|\alpha_{1}(s)\right| d s\right)^{p} \quad \forall h \in\left[0, T-t_{0}\right]
$$

Proof of Lemma 2.3: $\quad$ Let $\gamma=(B / A)^{1 / p}$. If $z_{1}\left(t_{0}+h\right)=z\left(t_{0}+h\right)$, then from the definition of $z_{1}$ we have

$$
B \leq A\left(z\left(t_{0}+h\right) / h\right)^{p}=A\left(z_{1}\left(t_{0}+h\right) / h\right)^{p}
$$

and therefore

$$
\int_{t_{0}}^{t_{0}+h}\left|\alpha_{1}(s)\right|^{p} d s \leq \int_{t_{0}}^{t_{0}+h}|\alpha(s)|^{p} d s \leq \frac{A}{h^{p-1}}\left(z\left(t_{0}+h\right)\right)^{p}+B h \leq \frac{2 A}{h^{p-1}}\left(z_{1}\left(t_{0}+h\right)\right)^{p}
$$


If on the contrary $z_{1}\left(t_{0}+h\right)>z\left(t_{0}+h\right)$, then there is some $h_{1}<h$ such that $z_{1}\left(t_{0}+h_{1}\right)=z\left(t_{0}+h_{1}\right)$ and $z_{1}(s)=\gamma\left(s-t_{0}\right)$ on $\left[t_{0}+h_{1}, t_{0}+h\right]$. Then we have from the previous step

$$
\begin{aligned}
\int_{t_{0}}^{t_{0}+h}\left|\alpha_{1}(s)\right|^{p} d s & =\int_{t_{0}}^{t_{0}+h_{1}}\left|\alpha_{1}(s)\right|^{p} d s+\int_{t_{0}+h_{1}}^{t_{0}+h}\left|\alpha_{1}(s)\right|^{p} d s \\
& \leq \frac{2 A}{h_{1}^{p-1}}\left(z_{1}\left(t_{0}+h_{1}\right)\right)^{p}+\left(h-h_{1}\right) \gamma^{p} \\
& \leq 2 A \gamma^{p} h_{1}+\left(h-h_{1}\right) \gamma^{p} \\
& \leq \frac{2 A}{h^{p-1}}\left(z_{1}\left(t_{0}+h\right)\right)^{p}
\end{aligned}
$$

Next we show - in a kind of reverse Hölder inequality - that if a map satisfies the inequality given by Lemma 2.3, then it is "more integrable" than what we could expect. There are several results of this nature in the literature since Gehring seminal work [5] (see for instance [4] and the references therein).

Lemma 2.4 Let $A>1$ and $p>1$. Then there are constants $\theta=\theta(A, p)>p$ and $C=C(A, p)>0$ such that, for any $\alpha \in L^{p}(0,1)$ such that

$$
\frac{1}{h} \int_{0}^{h}|\alpha(s)|^{p} d s \leq A\left(\frac{1}{h} \int_{0}^{h}|\alpha(s)| d s\right)^{p} \quad \forall h \in[0,1],
$$

we have

$$
\int_{0}^{h}|\alpha(s)| d s \leq C\|\alpha\|_{p} h^{1-1 / \theta} \quad \forall h \in[0,1] .
$$

Moreover, the optimal choice of $\theta$ is such that $\gamma=1-1 / \theta$ is the smallest root of $\varphi(s)=s^{p}-A(1-p+p s)$.

A possible proof of the Lemma is the following: using Gehring's result we can show that a map $\alpha$ satisfying (8) belongs in some $L^{r}$ for some $r>p$ with a $L^{r}$ norm controlled by its $L^{p}$ norm, and then use Hölder inequality. We have choosen to present in Appendix a new and direct proof using a completely different approach.

Combining Lemma 2.2, Lemma 2.3 and Lemma 2.4 we get:

Lemma 2.5 There are constants $\theta>p$ and $C$ depending only on $M, \delta, p, T$ such that, for any $x_{0} \in \mathbb{R}^{N}$ and any $t_{0}<T$, if $\bar{x}$ is optimal for the initial position $x_{0}$ at time $t_{0}$, then

$$
\int_{t_{0}}^{t_{0}+h}\left|\bar{x}^{\prime}(s)\right| d s \leq C\left(T-t_{0}\right)^{1 / \theta-1 / p} h^{1-1 / \theta} \quad \forall h \in\left[t_{0}, T\right]
$$


Proof of Lemma 2.5 : Let $\bar{x}$ be optimal for $\left(x_{0}, t_{0}\right)$. From Lemma 2.2 we know that

$$
\frac{1}{h} \int_{t_{0}}^{t_{0}+h}\left|\bar{x}^{\prime}(s)\right|^{p} d s \leq A\left(\frac{1}{h} \int_{t_{0}}^{t_{0}+h}\left|\bar{x}^{\prime}(s)\right| d s\right)^{p}+B \quad \forall h \in\left[0, T-t_{0}\right]
$$

for some constants $A, B$ depending only on $M, \delta, T$ and $p$. Setting $\alpha(t)=$ $\left|\bar{x}^{\prime}(t)\right|, z(t)=\int_{t_{0}}^{t} \alpha(s) d s, z_{1}(t)=\max \left\{z(t),(B / A)^{1 / p}\left(t-t_{0}\right)\right\}$ and $\alpha_{1}(t)=$ $z_{1}^{\prime}(t)$, we have from Lemma 2.3 .

$$
\frac{1}{h} \int_{t_{0}}^{t_{0}+h}\left(\alpha_{1}(s)\right)^{p} d s \leq 2 A\left(\frac{1}{h} \int_{t_{0}}^{t_{0}+h} \alpha_{1}(s) d s\right)^{p} \quad \forall h \in\left[0, T-t_{0}\right]
$$

Applying Lemma 2.4 to the constants $p$ and $2 A$ and with a proper scalling, we get that there exists $\theta>p$ and $C_{2}$ depending only on $M, \delta, T$ and $p$ such that

$$
\int_{t_{0}}^{t_{0}+h}\left|\bar{x}^{\prime}(s)\right| d s \leq \int_{t_{0}}^{t_{0}+h} \alpha_{1}(s) d s \leq\left(T-t_{0}\right)^{\frac{1}{\theta}-\frac{1}{p}} C_{2}\left\|\alpha_{1}\right\|_{p} h^{1-1 / \theta}
$$

where we can estimate $\left\|\alpha_{1}\right\|_{L^{p}\left(\left[t_{0}, T\right]\right)}$ as follows: Let

$$
\bar{t}=\max \left\{t \in\left[t_{0}, T\right] \mid z_{1}(t)=z(t)\right\} .
$$

Then

$$
\int_{t_{0}}^{T} \alpha_{1}^{p}(s) d s=\int_{t_{0}}^{\bar{t}} \alpha_{1}^{p}(s) d s+\int_{\bar{t}}^{T}\left(\frac{B}{A}\right)^{p} d s \leq \int_{t_{0}}^{\bar{t}} \alpha^{p}(s) d s+\left(\frac{B}{A}\right)^{p}(T-\bar{t})
$$

where, from Lemma 2.1, we have

$$
\int_{t_{0}}^{\bar{t}} \alpha^{p}(s) d s \leq K
$$

Therefore $\left\|\alpha_{1}\right\|_{p} \leq C_{3}$, where $C_{3}=C_{3}(M, \delta, p, T)$ and the proof is complete.

QED

\section{Regularity of the value function}

We are now ready to prove Theorem 1.1 . 
Space regularity : Let $x_{0}, x_{1} \in \mathbb{R}^{N}, t_{0}<T$. We assume that

$$
\left|x_{1}-x_{0}\right| \leq C \frac{(1-p / \theta)}{p-1}\left(T-t_{0}\right)^{1-1 / p} \wedge 1
$$

where $C$ and $\theta$ are the constants which appear in Lemma 2.5. We claim that

$$
u\left(x_{1}, t_{0}\right)-u\left(x_{0}, t_{0}\right) \leq K_{1}\left(T-t_{0}\right)^{-(p-1)(\theta-p) /(p(\theta-1))}\left|x_{1}-x_{0}\right|^{(\theta-p) /(\theta-1)}
$$

where $K_{1}=K_{1}(M, p, T, \delta)$.

Indeed, let $\bar{x}$ be an optimal trajectory for $\left(x_{0}, t_{0}\right)$. For $h \in\left(0, T-t_{0}\right)$ let

$$
\tilde{x}(t)= \begin{cases}\frac{\bar{x}\left(t_{0}+h\right)-x_{1}}{h}\left(t-t_{0}\right)+x_{1} & \text { if } t \in\left[t_{0}, t_{0}+h\right] \\ \bar{x}(t) & \text { otherwise }\end{cases}
$$

From Lemma 2.5 we have

$$
\left|\bar{x}\left(t_{0}+h\right)-x_{0}\right| \leq \int_{t_{0}}^{t_{0}+h}\left|\bar{x}^{\prime}(s)\right| d s \leq C\left(T-t_{0}\right)^{1 / \theta-1 / p} h^{1-1 / \theta} .
$$

Therefore, since $\tilde{x}(T)=\bar{x}(T)$, we have

$$
\begin{aligned}
u\left(x_{1}, t_{0}\right) & \leq \int_{t_{0}}^{T} a(\tilde{x}(s), s)\left|f(\tilde{x}(s), s)+\tilde{x}^{\prime}(s)\right|^{p} d s+g(\tilde{x}(T)) \\
& \leq u\left(x_{0}, t_{0}\right)+\int_{t_{0}}^{t_{0}+h} a(\tilde{x}(s), s)\left|f(\tilde{x}(s), s)+\tilde{x}^{\prime}(s)\right|^{p} d s \\
& \leq u\left(x_{0}, t_{0}\right)+M 2^{p-1}\left(M^{p} h+h^{1-p}\left|\bar{x}\left(t_{0}+h\right)-x_{1}\right|^{p}\right) \\
& \leq u\left(x_{0}, t_{0}\right)+M 2^{p-1}\left(M^{p} h+h^{1-p}\left(\left|\bar{x}\left(t_{0}+h\right)-x_{0}\right|+\left|x_{0}-x_{1}\right|\right)^{p}\right. \\
& \leq u\left(x_{0}, t_{0}\right)+M 2^{p-1}\left(M^{p} h+h^{1-p}\left(C_{0} h^{1-1 / \theta}+\left|x_{0}-x_{1}\right|\right)^{p}\right.
\end{aligned}
$$

where we have set $C_{0}=C\left(T-t_{0}\right)^{1 / \theta-1 / p}$. Choosing

$$
h=\left(\frac{1}{C_{0}} \frac{p-1}{1-p / \theta}\left|x_{0}-x_{1}\right|\right)^{\theta /(\theta-1)}
$$

we have $h \leq\left(T-t_{0}\right)$ (from assumption (9)) and therefore

$$
u\left(x_{1}, t_{0}\right)-u\left(x_{0}, t_{0}\right) \leq K_{1}^{\prime}\left(T-t_{0}\right)^{-(\theta-p)(p-1) /(p(\theta-1))}\left|x_{1}-x_{0}\right|^{(\theta-p) /(\theta-1)}
$$

where $K_{1}^{\prime}=K_{1}^{\prime}(M, \delta, p, T)$. Whence (10).

Time regularity : Let $x_{0}$ be fixed and $t_{0}<t_{1}<T-\tau$. We assume that

$$
t_{1}-t_{0} \leq K_{3} \tau^{(2 \theta p-p-\theta) /(p(\theta-1))}
$$


for some constant $K_{3}=K_{3}(M, \delta, p, T)$ to be fixed later, where $\theta$ is given by Lemma (2.5). We claim that

$$
\left|u\left(x_{0}, t_{0}\right)-u\left(x_{0}, t_{1}\right)\right| \leq K_{2} \tau^{-(\theta-p) / \theta}\left(t_{1}-t_{0}\right)^{(\theta-p) / \theta}
$$

for some constant $K_{2}=K_{2}(M, \delta, p, T)$.

Indeed, let $\bar{x}$ be optimal for $\left(x_{0}, t_{1}\right)$. Then setting

$$
\tilde{x}(t)= \begin{cases}x_{0} & \text { if } t \in\left[t_{0}, t_{1}\right] \\ \bar{x}(t) & \text { otherwise }\end{cases}
$$

we have

$$
\begin{aligned}
u\left(x_{0}, t_{0}\right) & \leq \int_{t_{0}}^{t_{1}} a\left(x_{0}, s\right)\left|f\left(x_{0}, s\right)\right|^{p} d s+u\left(x_{0}, t_{1}\right) \\
& \leq M^{p+1}\left|t_{1}-t_{0}\right|+u\left(x_{0}, t_{1}\right)
\end{aligned}
$$

which gives the desired inequality provided $K_{2}$ is sufficiently large.

To get a reverse inequality, let $\bar{x}$ be now optimal for $\left(x_{0}, t_{0}\right)$. Using Lemma 2.5 we have that

$$
\begin{aligned}
\left|\bar{x}\left(t_{1}\right)-x_{0}\right| & \leq \int_{t_{0}}^{t_{1}}\left|\bar{x}^{\prime}(s)\right| d s \leq C\left(T-t_{0}\right)^{1 / \theta-1 / p}\left(t_{1}-t_{0}\right)^{1-1 / \theta} \\
& \leq C \frac{(1-p / \theta)}{p-1}\left(T-t_{1}\right)^{1-1 / p} \wedge 1
\end{aligned}
$$

from the choice of $t_{1}-t_{0}$ in (11) and $K_{3}$ sufficiently small. Note that we have

$$
u\left(\bar{x}\left(t_{1}\right), t_{1}\right) \leq u\left(x_{0}, t_{0}\right)-\int_{t_{0}}^{t_{1}} a(\bar{x}(s), s)\left|f(\bar{x}(s), s)+\bar{x}^{\prime}(s)\right|^{p} d s \leq u\left(x_{0}, t_{0}\right)
$$

Hence, using the space regularity of $u$ (recall that (12) holds) we get

$$
\begin{aligned}
u\left(x_{0}, t_{1}\right) & \leq u\left(x_{0}, t_{1}\right)-u\left(\bar{x}\left(t_{1}\right), t_{1}\right)+u\left(x_{0}, t_{0}\right) \\
& \leq u\left(x_{0}, t_{0}\right)+K_{1} C\left(T-t_{1}\right)^{-(p-1)(\theta-p) /(p(\theta-1))}\left|\bar{x}\left(t_{1}\right)-x_{0}\right|^{(\theta-p) /(\theta-1)} \\
& \leq u\left(x_{0}, t_{0}\right)+K_{2} \tau^{-(\theta-p) / \theta}\left(t_{1}-t_{0}\right)^{(\theta-p) / \theta}
\end{aligned}
$$

QED

\section{Appendix : proof of Lemma 2.4}

We note later use that the map $\varphi(s)=s^{p}-A(1-p+p s)$ has two roots, the smallest one - denoted by $\gamma$-belonging to the interval $\left(1-1 / p, A^{1 /(p-1)}\right)$, the other one being larger than $A^{1 /(p-1)}$. Moreover, if $\varphi(s) \leq 0$, then $s \geq \gamma$.

Let

$$
\mathcal{E}=\left\{\alpha \in L^{p}(0,1), \alpha \geq 0, \alpha \text { satisfies (8) and }\|\alpha\|_{p} \leq 1\right\}
$$


We note that $\mathcal{E}$ is convex, closed and bounded in $L^{p}(0,1)$. Therefore the problem

$$
\xi(\tau)=\max \left\{\int_{0}^{\tau} \alpha(s) d s, \alpha \in \mathcal{E}\right\}
$$

has a unique maximum denoted $\bar{\alpha}_{\tau}$ for any $\tau \in(0,1]$ (uniqueness comes from the fact that inequality (8) is positively homogeneous, which entails that at the optimum inequality $\|\alpha\|_{p} \leq 1$ is an equality).

In order to prove the Lemma, we only need to show that

$$
\xi(\tau) \leq C \tau^{\gamma} \quad \forall \tau \in[0,1]
$$

for a suitable choice of $C$, because again inequality (8) is positively homogeneous in $\alpha$.

The proof of (13) is achieved in two steps. In the first one, we explain the structure of the optima. Then we deduce from this that $\xi$ satisfies a differential equation, which gives the desired bound.

Structure of the optima : We claim that there is some $\bar{\tau}>0$ such that for any $\tau \in(0, \bar{\tau})$,

$$
\bar{\alpha}_{\tau}(t)= \begin{cases}a_{\tau} & \text { on }[0, \tau) \\ b_{\tau} & \text { on }\left[\tau, \tau_{1}\right) \\ A^{-1 / p} \gamma t^{\gamma-1} & \text { on }\left[\tau_{1}, 1\right]\end{cases}
$$

where $0<b_{\tau} \leq a_{\tau}$ and $\tau<\tau_{1}<1$.

Proof of the claim : Let $\bar{x}_{\tau}(t)=\int_{0}^{t} \bar{\alpha}_{\tau}(s) d s$. To show that $\bar{\alpha}_{\tau}$ is constant on $[0, \tau)$, we introduce the map $\alpha(s)=\frac{\bar{x}_{\tau}(\tau)}{\tau}$ on $[0, \tau), \alpha=\bar{\alpha}_{\tau}$ otherwise. Then $\alpha$ belongs to $\mathcal{E}$ and is also optimal. Hence $\alpha=\bar{\alpha}_{\tau}$, which shows that $\bar{\alpha}_{\tau}$ is constant on $[0, \tau)$.

With similar arguments we can prove that, if there is a strict inequality in (8) for $\bar{\alpha}_{\tau}$ at some $h \geq \tau$, then $\bar{\alpha}_{\tau}$ is locally constant in a neighbourhood of $h$ in $[\tau, 1]$. In particular, since $\bar{\alpha}_{\tau}$ is constant on $[0, \tau)$, inequality (8) is strict for $\bar{\alpha}_{\tau}$ at $\tau$, and there is a maximal interval $\left[\tau, \tau_{1}\right)$ on which $\bar{\alpha}_{\tau}$ is constant. We set $a_{\tau}=\bar{\alpha}_{\tau}\left(0^{+}\right)$and $b_{\tau}=\bar{\alpha}_{\tau}\left(\tau^{+}\right)$.

In order to show that $a_{\tau} \geq b_{\tau}$, we prove that

$$
\text { the map } t \rightarrow \bar{x}_{\tau}(t) / t \text { is nonincreasing. }
$$

Indeed, let $t>0$ be fixed and $x(s)=\max \left\{\bar{x}_{\tau}(s), \frac{\bar{x}_{\tau}(t)}{t} s\right\}$ if $s \in[0, t]$ and $x=\bar{x}_{\tau}$ otherwise. Let us check that $x^{\prime}$ is admissible and optimal. Let 
$I \subset(0, t)$ be the open set $\left\{x>\bar{x}_{\tau}\right\}$. We can write $I$ as the (at most) enumerable union of disjoint intervals $\left(c_{i}, d_{i}\right)$. Since $x$ is affine on each interval $\left(c_{i}, d_{i}\right)$ with $x\left(c_{i}\right)=\bar{x}\left(c_{i}\right)$ and $x\left(d_{i}\right)=\bar{x}\left(d_{i}\right)$ we have

$$
\int_{c_{i}}^{d_{i}}\left|x^{\prime}\right|^{p} \leq \int_{c_{i}}^{d_{i}}\left|\bar{x}_{\tau}^{\prime}\right|^{p} \quad \forall i
$$

Since $x^{\prime}=\bar{x}_{\tau}$ a.e. in $[0,1] \backslash I$, we get $\left\|x^{\prime}\right\|_{p} \leq\left\|\bar{x}_{\tau}^{\prime}\right\|_{p}=1$. Moreover, for any $h>0$ such that $x(h)=\bar{x}_{\tau}(h)$, (15) and the admissibility of $\bar{x}_{\tau}^{\prime}$ also give

$$
\frac{1}{h} \int_{0}^{h}\left|x^{\prime}\right|^{p} \leq \frac{1}{h} \int_{0}^{h}\left|\bar{x}_{\tau}^{\prime}\right|^{p} \leq A\left(\frac{1}{h} \bar{x}_{\tau}(h)\right)^{p}=A\left(\frac{1}{h} x(h)\right)^{p} .
$$

If $x(h)>\bar{x}_{\tau}(h)$, let $h_{1}=\max \left\{s \leq h \mid x(s)=\bar{x}_{\tau}(s)\right\}$. Then $x(s)=\frac{\bar{x}_{\tau}(t)}{t} s$ on $\left[h_{1}, h\right]$ and so

$$
\begin{aligned}
\frac{1}{h} \int_{0}^{h}\left|x^{\prime}\right|^{p} & =\frac{1}{h} \int_{0}^{h_{1}}\left|x^{\prime}\right|^{p}+\frac{1}{h} \int_{h_{1}}^{h}\left|x^{\prime}\right|^{p} \\
& \leq \frac{A h_{1}}{h}\left(\frac{1}{h_{1}} x\left(h_{1}\right)\right)^{p}+\frac{1}{h}\left(h-h_{1}\right)\left(\frac{\bar{x}_{\tau}(t)}{t}\right)^{p} \\
& \leq \frac{A h_{1}}{h}\left(\frac{\bar{x}_{\tau}(t)}{t}\right)^{p}+A \frac{1}{h}\left(h-h_{1}\right)\left(\frac{\bar{x}_{\tau}(t)}{t}\right)^{p} \\
& \leq A\left(\frac{\bar{x}_{\tau}(t)}{t}\right)^{p}=A\left(\frac{1}{h} x(h)\right)^{p}
\end{aligned}
$$

So $x^{\prime}$ is admissible. Since $x(\tau) \geq \bar{x}_{\tau}(\tau), x$ is also optimal. So $x=\bar{x}_{\tau}$ and (14) is proved.

Note that (14) implies that $a_{\tau} \geq b_{\tau}$ and

$$
\frac{s \bar{x}_{\tau}^{\prime}(s)}{\bar{x}_{\tau}(s)} \leq 1<A^{1 /(p-1)} \quad \text { for a.e. } s \in[0,1] .
$$

Let us now assume that $\tau_{1}<1$. To prove that $\bar{\alpha}_{\tau}(s)=A^{-1 / p} \gamma s^{\gamma-1}$ on $\left[\tau_{1}, 1\right]$, we show that there is an equality in (8) for $\bar{\alpha}_{\tau}$ on $\left[\tau_{1}, 1\right]$. Indeed, otherwise, $\bar{\alpha}_{\tau}$ is constant on some maximal interval $(u, v)$ with $\tau_{1} \leq u<v \leq$ 1. We note that equality holds in (8) at $u$ because $\bar{\alpha}_{\tau}$ is not locally constant at this point. Taking the derivative with respect to $h$ in (8) at $u$ we get

$$
\left(\bar{\alpha}_{\tau}\left(u^{+}\right)\right)^{p} \leq-\frac{(p-1) A}{u^{p}}\left(\bar{x}_{\tau}(u)\right)^{p}+\frac{p A}{u^{p-1}}\left(\bar{x}_{\tau}(u)\right)^{p-1} \bar{\alpha}_{\tau}\left(u^{+}\right),
$$

i.e.,

$$
\left(\frac{u \bar{\alpha}_{\tau}\left(u^{+}\right)}{\bar{x}_{\tau}(u)}\right)^{p}-A\left(1-p+p \frac{u \bar{\alpha}_{\tau}\left(u^{+}\right)}{\bar{x}_{\tau}(u)}\right) \leq 0 .
$$


From the analysis of $\varphi$, this implies that

$$
\bar{\alpha}_{\tau}\left(u^{+}\right) \geq \gamma \frac{\bar{x}_{\tau}(u)}{u} .
$$

Let us define

$x(s)=\bar{x}_{\tau}(s)$ on $[0, u], x(s)=\frac{\bar{x}_{\tau}(u)}{u^{\gamma}} s^{\gamma}$ on $[u, v], x(s)=\frac{x\left(v^{-}\right)}{\bar{x}_{\tau}(v)} \bar{x}_{\tau}(s)$ on $[v, 1]$

and $\alpha=x^{\prime}$. Since $\alpha\left(u^{+}\right)=\gamma \frac{\bar{x}_{\tau}(u)}{u} \leq \bar{\alpha}_{\tau}\left(u^{+}\right)$, one easily checks that $x \leq \bar{x}_{\tau}$ and $\alpha \leq \bar{\alpha}_{\tau}$ on $[0,1]$. Moreover, a straightforward verification shows that $x$ satisfies (8). Hence $x$ is also optimal, which is impossible. So there is an equality in (8) for $\bar{\alpha}_{\tau}$ on $\left[\tau_{1}, 1\right]$. Taking the derivative in this equality shows that $\bar{\alpha}_{\tau}$ solves

$$
\bar{\alpha}_{\tau}^{p}(s)=-\frac{p A}{s^{p-1}}\left(\bar{x}_{\tau}(s)\right)^{p}+\frac{A}{s^{p}}\left(\bar{x}_{\tau}(s)\right)^{p-1} \bar{\alpha}_{\tau}(s) \quad \text { on }\left[\tau_{1}, 1\right] .
$$

From (16) and the analysis of $\varphi$, this implies that $\bar{x}_{\tau}^{\prime}(s)=\gamma \frac{\bar{x}_{\tau}(s)}{s}$ on $\left[\tau_{1}, 1\right]$. Hence $\bar{x}_{\tau}(s)=C s^{\gamma}$ for some constant $C$. Since there is an equality in (8) at $h=1$ and since $\left\|\bar{\alpha}_{\tau}\right\|_{p}=1,1=A\left(\bar{x}_{\tau}(1)\right)^{p}$ and therefore $C=A^{-1 / p}$.

Finally we have to show that $\tau_{1}<1$ for any $\tau \in(0, \bar{\tau})$. Indeed, assume otherwise that $\tau_{1}=1$ for arbitrary small $\tau$. Since $x(t)=A^{-1 / p} t^{\gamma}$ is admissible, we have $a_{\tau} \tau \geq A^{-1 / p} \tau^{\gamma}$. Hence $a_{\tau} \rightarrow+\infty$ as $\tau \rightarrow 0^{+}$. Moreover the constraint $\left\|\bar{\alpha}_{\tau}\right\|_{p}=1$ implies that $b_{\tau}$ is bounded when $\tau \rightarrow 0^{+}$. Hence, for any $k$ large, we can find $\tau>0$ such that $a_{\tau}>k b_{\tau}$. Writing inequality (8) at $h=k \tau$ then gives

$a_{\tau}^{p} \tau \leq \frac{A}{(1+k)^{p-1} \tau^{p-1}}\left(a_{\tau} \tau+b_{\tau} k \tau\right)^{p} \leq \frac{A}{(1+k)^{p-1} \tau^{p-1}}\left(2 a_{\tau} \tau\right)^{p}=\frac{2^{p} A}{(1+k)^{p-1}} a_{\tau}^{p} \tau$

whence a contradiction since $k$ is arbitrarily large.

A differential equation for $\xi$ : To complete the proof of (13), we are going to show that $\xi$ is locally Lipschitz continous and satisfies

$$
(-\tau) \xi^{\prime}(\tau)+\gamma \xi(\tau)=0 \quad \text { for a.e. } \tau \in(0, \bar{\tau}) .
$$

From this (13) follows easily for a suitable choice of $C$.

Proof of (17) : Let us extend the optimal solutions by $A^{-1 / p} \gamma s^{\gamma-1}$ on $[1,+\infty)$ for $\tau \in(0, \bar{\tau})$. For $\lambda>0$, let

$$
\alpha_{\lambda \tau}(s)=\bar{\alpha}_{\tau}(\lambda s) \quad s \geq 0 .
$$


Then $\alpha_{\lambda \tau}$ satisfies (8) and

$$
\left\|\alpha_{\lambda \tau}\right\|_{p}=\lambda^{-1 / p}\left(1+\int_{1}^{\lambda} \bar{\alpha}_{\tau}^{p}\right)^{1 / p}
$$

Hence $\alpha_{\lambda \tau} /\left\|\alpha_{\lambda \tau}\right\|_{p}$ is admissible and

$$
\xi\left(\frac{\tau}{\lambda}\right) \geq \frac{\int_{0}^{\tau / \lambda} \alpha_{\lambda \tau}}{\left\|\alpha_{\lambda \tau}\right\|_{p}}=\frac{\lambda^{1 / p-1} \xi(\tau)}{\left(1+\int_{1}^{\lambda} \bar{\alpha}_{\tau}^{p}\right)^{1 / p}} .
$$

with an equality for $\lambda=1$. In particular, this shows that $\xi$ is locally Lipschitz continuous in $(0,1]$. Moreover, at each point $\tau$ at which $\xi$ has a derivative, we have, by taking the derivative with respect to $\lambda$ at $\lambda=1$ in (18):

$$
(-\tau) \xi^{\prime}(\tau)=(1 / p-1) \xi(\tau)-\frac{\xi(\tau)}{p} \bar{\alpha}_{\tau}^{p}(1)=\xi(\tau)\left(1 / p-1-A \gamma^{p} / p\right)=-\gamma \xi(\tau)
$$

on $(0, \bar{\tau})$. Whence (17).

QED

\section{References}

[1] Bardi M., Capuzzo Dolcetta I. Optimal Control And viscosity SOlutions of HAMILTON-JACOBI-BELlman EQUATIONS. Birkhäuser, 1996.

[2] Barles G. Solutions de viscosité des Équations de Hamilton-Jacobi. Springer-Verlag, Berlin, 1994.

[3] Barles, G. Regularity results for first order Hamilton-Jacobi equations. Differential Integral Equations 3 (1990), no. 1, 103125.

[4] Bensoussan, A., Frehse, J. Regularity Results for NonlinEAR ELLIPTIC SYSTEMS AND APPliCATiOns. Applied Mathematical Sciences, 151. Springer-Verlag, Berlin, 2002.

[5] Gehring F.W., The $L^{p}$-integrability of the partial derivatives of a quasiconformal mapping. Acta Math. 130 (1973), 265-277.

[6] Lions, P.-L. Regularizing effects for first-order Hamilton-Jacobi equations. Applicable Anal. 20 (1985), no. 3-4, 283-307. 
[7] Rampazzo, F., Sartori, C. Hamilton-Jacobi-Bellman equations with fast gradient-dependence. Indiana Univ. Math. J. 49 (2000), no. 3, 1043-1077. 